\title{
Equivalent Cardioprotection Induced by Ischemic and Hypoxic Preconditioning
}

\author{
Xujin Xiang ${ }^{1}$ Haixia Lin ${ }^{2}$ Jin Liu ${ }^{1}$ Zeyan Duan ${ }^{1}$ \\ ${ }^{1}$ Department of Anesthesiology, West China Hospital, Sichuan \\ University, Chengdu, China \\ ${ }^{2}$ Laboratory of Anesthesiology and Critical Care Medicine, West China \\ Hospital, Sichuan University, Chengdu, China
}

\begin{abstract}
Address for correspondence Jin Liu, MD, Department of Anesthesiology, West China Hospital, Sichuan University, No. 37 Wai Nan Guo Xue Xiang, Chengdu 610041, Sichuan, China (e-mail: scujinliu@gmail.com).
\end{abstract}

Thorac Cardiovasc Surg 2013;61:229-233.

\begin{abstract}
Keywords

- ischemic preconditioning

- hypoxic preconditioning

- reperfusion injury

Objective We aimed to compare cardioprotection induced by various hypoxic preconditioning (HPC) and ischemic preconditioning (IPC) protocols.

Methods Isolated rat hearts were randomly divided into 7 groups ( $n=7$ per group) and received 3 or 5 cycles of 3-minute ischemia or hypoxia followed by 3-minute reperfusion (IPC33 or HPC33 or IPC53 or HPC53 group), 3 cycles of 5-minute ischemia or hypoxia followed by 5-minute reperfusion (IPC35 group or HPC35 group), or 30-minute perfusion (ischemic/reperfusion group), respectively. Then all the hearts were subjected to 50-minute ischemia and 120-minute reperfusion. Cardiac function, infarct size, and coronary flow rate (CFR) were evaluated.

Results Recovery of cardiac function and CFR in IPC 35, HPC35, and HPC53 groups was significantly improved as compared with I/R group $(p<0.01)$. There were no significant differences in cardiac function parameters between IPC35 and HPC35 groups. Consistently, infarct size was significantly reduced in IPC35, HPC35, and HPC53 groups compared with ischemic/reperfusion group.

Conclusion Multiple-cycle short duration HPC exerted cardioprotection, which was as powerful as that of IPC.
\end{abstract}

\section{Introduction}

Ischemic/reperfusion ( $\mathrm{I} / \mathrm{R})$ injury is closely related to organ transplantation, stroke, cardiovascular surgery, and disease. Myocardial $\mathrm{I} / \mathrm{R}$ injury is the major reason of death associated with heart desease. ${ }^{1}$ Ischemic preconditioning (IPC), consisting of single or multiple episodes of sublethal ischemia and reperfusion, markedly reduces myocardial infarct size and is the most effective endogenous protective approach against $\mathrm{I} / \mathrm{R}$ injury. However, clinical application of IPC is limited for practical reasons and high risks. ${ }^{2}$ New ways against I/R injury are urgently required.

Hypoxic preconditioning (HPC) is a phenomenon that sublethal hypoxia increases cellular resistance against following lethal I/R injury. The clinical application of HPC may be much easier and safer than that of IPC, for hypoxia was not accompanied by blood flow cessation. HPC has been verified in various tissues. However, most studies have been focused on prolonged HPC, which requires hours to weeks, or delayed HPC, in which the protective effect occurs approximately 24 hours after the preconditioning stimulus. ${ }^{3-6}$ Early cardioprotection, which occurs immediately after the preconditioning induced by short duration HPC, would be of great value clinically. Only several studies demonstrated that one-cycle short duration HPC (5-minute hypoxia followed by 10-minute reperfusion) exerted early cardioprotection. ${ }^{7-9}$ Cai et al reported that 30 minutes after preconditioning of mice with 5 cycles of 6 -minute $6 \% \mathrm{O}_{2}$ and 6 -minute $21 \% \mathrm{O}_{2}$, the hearts were not protected against $\mathrm{I} / \mathrm{R}$ injury. ${ }^{10}$ It has not been known that whether multiple-cycle short duration HPC could produce early protective effects in received

December 8, 2011

accepted after revision

April 2, 2012

published online

September 7, 2012 (c) 2013 Georg Thieme Verlag KG Stuttgart · New York
DOI http://dx.doi.org/ 10.1055/s-0032-1322609. ISSN 0171-6425. 
isolated rat hearts. Therefore, we investigated the early effects of various cycles and the number and hypoxic duration of each cycle on isolated rat hearts subjected to I/R injury and compare the effects with cardioprotection by IPC.

\section{Materials and Methods}

\section{Animal and Rat Langendorff Model}

Hearts from adult male Sprague-Dawley rats (220-300 g) anesthetized with pentobarbital sodium ( $50 \mathrm{mg} / \mathrm{kg}$ intraperitoneally) were mounted in a Langendorff model (Radnoti, Monrovia, California, United States) and perfused with KrebsHenseleit buffer (in mM: $118.0 \mathrm{NaCl}, 4.7 \mathrm{KCl}, 2.5 \mathrm{CaCl}_{2}, 1.2$ $\mathrm{KH}_{2} \mathrm{PO}_{4}, 1.2 \mathrm{MgSO}_{4}, 25.0 \mathrm{NaHCO}_{3}, 11.1$ glucose, and 0.125 EDTA-2Na, pH: 7.35 to 7.45 ) at $37^{\circ} \mathrm{C}$ and a constant pressure of $100 \mathrm{~cm} \mathrm{H}_{2} \mathrm{O}$. Oxygenated $\mathrm{KH}$ buffer was continuously bubbled with $95 \% \mathrm{O}_{2}+5 \% \mathrm{CO}_{2}$. Hypoxic Krebs Henseleit $(\mathrm{KH})$ buffer was bubbled with $95 \% \mathrm{~N}_{2}+5 \% \mathrm{CO}_{2}$.

\section{Experimental Protocol}

After being stabilized with oxygenated $\mathrm{KH}$ buffer for $20 \mathrm{mi}-$ nutes, 49 isolated hearts were randomly divided into 7 groups ( $n=7$ per group) and received 3 cycles of 3-minute global ischemia or hypoxic KH buffer followed with 3-minute oxygenated $\mathrm{KH}$ buffer reperfusion (IPC33 or HPC33 group), 5 cycles of 3-minute global ischemia or hypoxic KH buffer followed with 3-minute oxygenated $\mathrm{KH}$ buffer reperfusion (IPC53 group or HPC53 group), 3 cycles of 5-minute global ischemia or hypoxic
KH buffer followed with 5-minute oxygenated KH buffer reperfusion (IPC35 group or HPC35 group), or 30-minute perfusion with oxygenated $\mathrm{KH}$ buffer (I/R group), respectively. Then all the hearts were subjected to 50-minute ischemia and 120-minute oxygenated $\mathrm{KH}$ buffer reperfusion. Cardiac left ventricle (LV) function, infarct size, and coronary flow rate (CFR) were evaluated. The experiment protocol was shown in - Fig. 1.

\section{Cardiac LV Function and CFR Measurement}

A water-filled latex balloon connected to a pressure transducer (Model SP844; MEMSCAP, Durham, North Carolina, United States) was inserted into the left ventricular cavity via the left auricle to measure cardiac LV function. Left ventricular developed pressure (LVDP, in $\mathrm{mm} \mathrm{Hg}$ ), its maximal and minimal rates of rise ([d $p / d t]$ max and $[\mathrm{d} p / \mathrm{d} t] \mathrm{min}$, in $\mathrm{mm} \mathrm{Hg} / \mathrm{s})$, and heart rate (HR) were measured at the end of stabilization (baseline), and at various times, during reperfusion. Coronary flow rate ( $\mathrm{mL} /$ minute) was also estimated at these time points. Recoveries of LVDP, $(\mathrm{d} p / \mathrm{d} t) \max ,(\mathrm{d} p / \mathrm{d} t) \mathrm{min}, \mathrm{HR}$, and CFR were, respectively, calculated as percentages of baseline values.

\section{Infarct Size Assessment}

At the end of 120 minutes of reperfusion, hearts were sliced into 2-mm transverse sections from apex to base. The slices were incubated at $37^{\circ} \mathrm{C}$ with $1 \%$ 2,3,5-triphenyltetrazolium chloride (TTC, Sigma-Aldrich, St. Louis, Missouri, United States) in phosphate buffer $(\mathrm{pH}=7.4)$ for 15 minutes and then fixed in a $4 \%$ paraformaldehyde phosphate buffer for 24

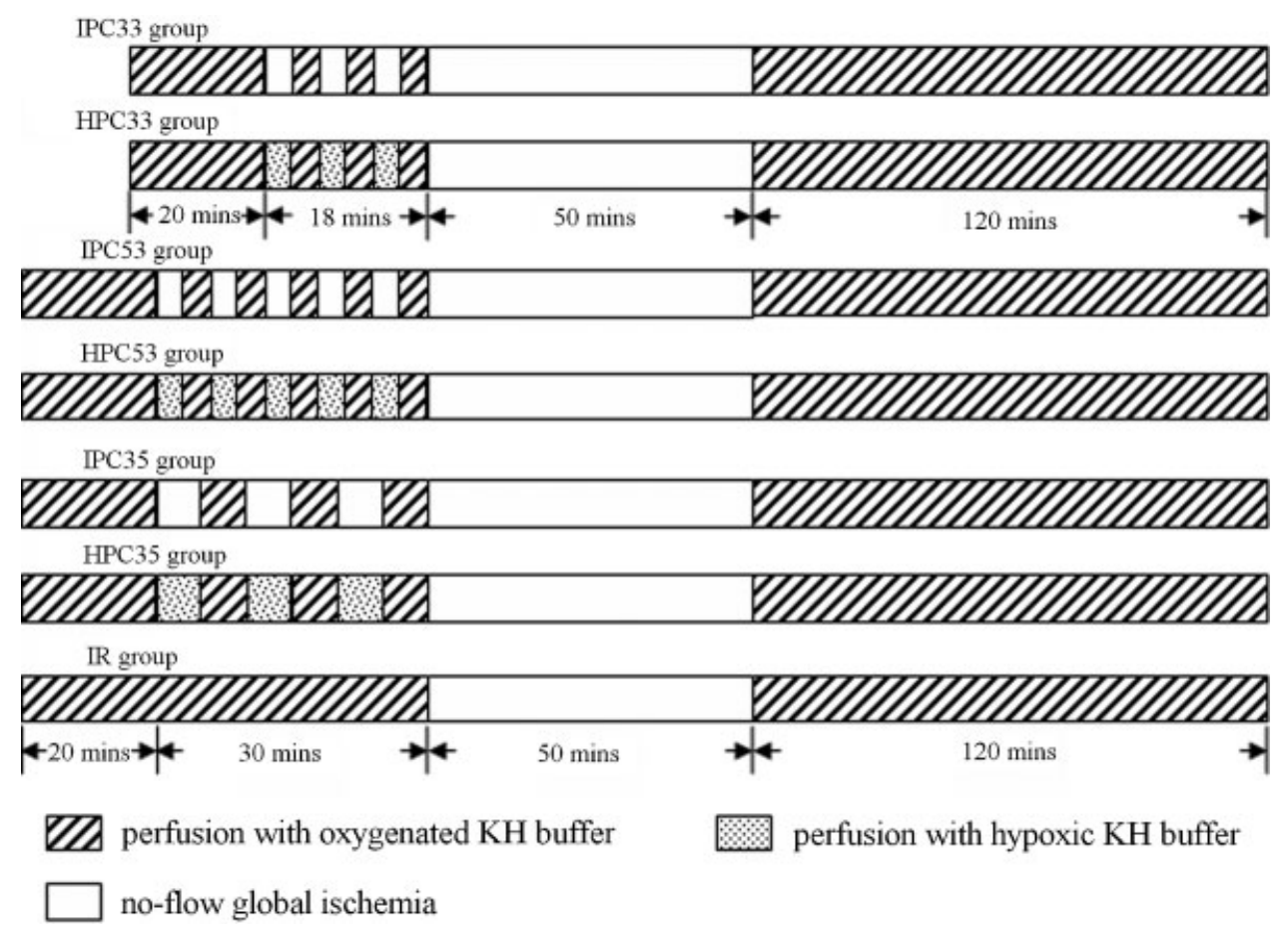

Fig. 1 The experimental protocol. After 20-minute equilibration, rat hearts received 3 cycles of 3-minute global ischemia or hypoxic KH buffer followed with 3-minute oxygenated KH buffer reperfusion (IPC33 group and HPC33 group), 5 cycles of 3-minute global ischemia or hypoxic KH buffer followed with 3-minute oxygenated KH buffer reperfusion (IPC53 group and HPC53 group), 3 cycles of 5-minute global ischemia or hypoxic KH buffer followed with 5-minute oxygenated KH buffer reperfusion (IPC35 group and HPC35 group), or 30-minute perfusion with oxygenated $\mathrm{KH}$ buffer (I/R group), respectively. Then all the hearts were subjected to 50 -minute ischemia and 120 -minute oxygenated $\mathrm{KH}$ buffer reperfusion.

Abbreviations: HPC, hypoxic preconditioning (HPC); IPC, ischemic preconditioning; IR, ischemic/reperfusion; KH, Krebs Henseleit. 

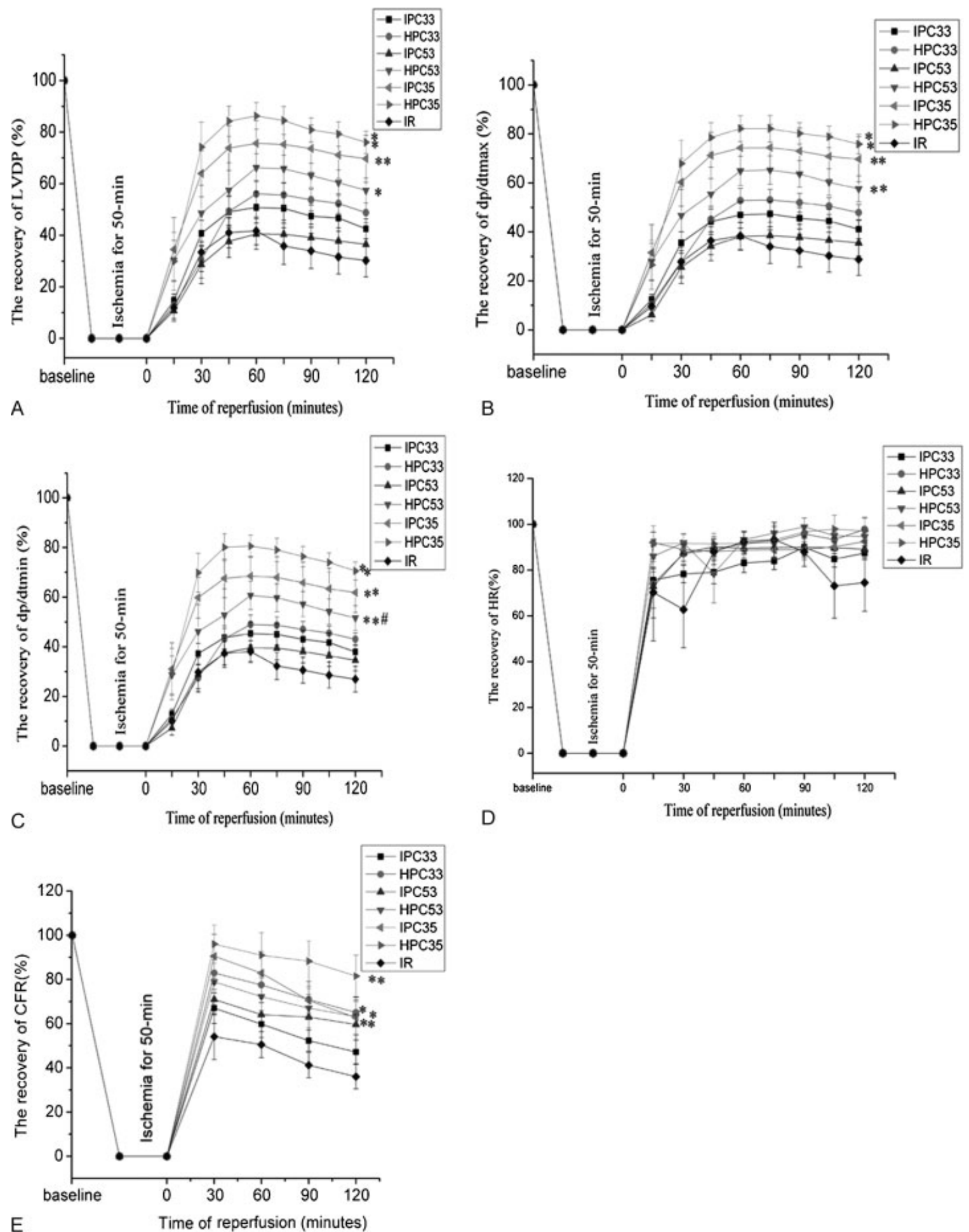

Fig. 2 Effects of IPC and HPC on left ventricle function of hearts subjected to ischemia/reperfusion injury. (A-E) The recovery of LVDP, (dp/dt)max and $(\mathrm{d} p / \mathrm{d} t) \mathrm{min}$, heart rate, and coronary flow rate during baseline and reperfusion. The zero time point demonstrated the beginning of reperfusion. Data are expressed as mean $\pm \mathrm{SE}, n=7 .{ }^{*} P<0.05,{ }^{* *} P<0.01$ compared with I/R group.

Abbreviations: HPC, hypoxic preconditioning; IPC, ischemic preconditioning; LVDP, left ventricular developed pressure; (dp/dt) max and (dp/dt) min, LVDP's maximal and minimal rates of rise.

hours. Subsequently, the slices were scanned (MRS-800P48U, MICROTEK, Shanghai, China). The infarct (white) area was assessed by planimetry using Image Pro Plus 5.0 software (Media Cybernetics, Silver Spring, Maryland, United States) and expressed as a percentage of total slice area.

\section{Statistical Analysis}

All values are expressed as means $\pm \mathrm{SE}$, the recovery of LVDP, $(\mathrm{d} p / \mathrm{d} t) \max ,(\mathrm{d} p / \mathrm{d} t) \min , \mathrm{HR}$, and CFR were analyzed by repeated-measures analysis of variance and using least significant difference procedure for post hoc test. Infarct size was 
tested with one-way analysis of variance followed by least significant difference procedure for post hoc test. All $p$ values less than 0.05 were considered statistically significant (version 16.0, SPSS, Chicago, Illinois, United States).

\section{Results}

\section{Recovery of Cardiac LV Function and CFR}

During reperfusion, recovery of LVDP, $(\mathrm{dp} / \mathrm{dt}) \max ,(\mathrm{dp} / \mathrm{dt})$ min, and CFR in IPC35, HPC35, and HPC53 groups were significantly higher than I/R group $(p<0.01)$. There was no significant difference in recovery of LVDP, (dp/dt)max, (dp/dt) min, or CFR between IPC35 and HPC35 groups. Recovery of LVDP, (dp/dt)max, and CFR did not differ significantly between HPC53 and HPC35 groups. Only recovery of (dp/dt) min in HPC53 group was lower than that in HPC35 group $(p<0.05)$ (-Fig. 2). There was no significant difference in HR among all the groups (data not shown; - Fig. 2E).

\section{Infarct Size}

Infarct size was significantly reduced in IPC35, HPC35, and HPC53 groups $(14.15 \pm 2.45 \%, 15.00 \pm 3.17 \%$, and $19.47 \pm$ $3.54 \%$, respectively) in comparison with $\mathrm{I} / \mathrm{R}$ group $(49.11 \pm 5.99 \%, p<0.01)$. There was no significant difference in infarct size among IPC35, HPC35, and HPC53 groups (-Fig. 3).

\section{Discussion}

In this study, our results demonstrated that multiple-cycle short duration HPC, which was performed by 3 cycles of 5 minute hypoxia followed with 5-minute reperfusion or 5 cycles of 3-minute hypoxic followed with 3-minute reperfusion, exerted early cardioprotection on isolated rat hearts subjected to I/R injury. The protection by multiple-cycle short duration HPC was comparable with that of IPC. Our finding was evidenced by improved recoveries of cardiac LV function and CFR and reduced myocardium infarct size.

Several studies have demonstrated that one-cycle IPC and HPC exerted similar cardioprotection. ${ }^{7-9}$ However, it has been unknown whether multiple-cycle HPC and IPC elicited similar cardioprotection. We demonstrated that early cardioprotection of multiple-cycle HPC was as powerful as that of IPC. In a wholebody hypoxia model, 30-minute intermittent hypoxia (repetitive cycles of 40 -second hypoxia with 5 or $10 \% \mathrm{O}_{2}$ followed by 20-second normoxia) did not induce delay cardioprotection in rat hearts. ${ }^{11}$ However, we indicated that 30 -minute intermittent HPC ( 3 cycles of 5-minute hypoxia followed with 5-minute reperfusion or 5 cycles of 3-minute hypoxic followed with 3-minute reperfusion) elicited early cardiac protection in isolated rat hears. Although Cai et al reported that 1 hour whole-body HPC ( 5 cycles of 6 minute hypoxia and 6 minute reoxygenation) could protect mice hearts against I/R injury 24 hours later but not 30 minutes later, ${ }^{10}$ we demonstrated that short duration HPC induced immediate cardioprotection.

The present result demonstrated that HPC53 ( 5 cycles of 3 minute hypoxia followed with 3 minute reperfusion) induced cardioprotection as robust as that of HPC35 (3 cycles of 5-

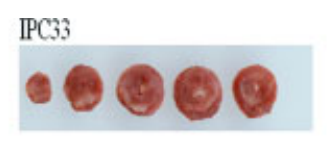

IPC53

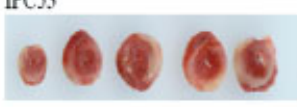

IPC35

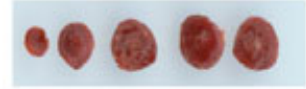

$\mathbb{R}$

A
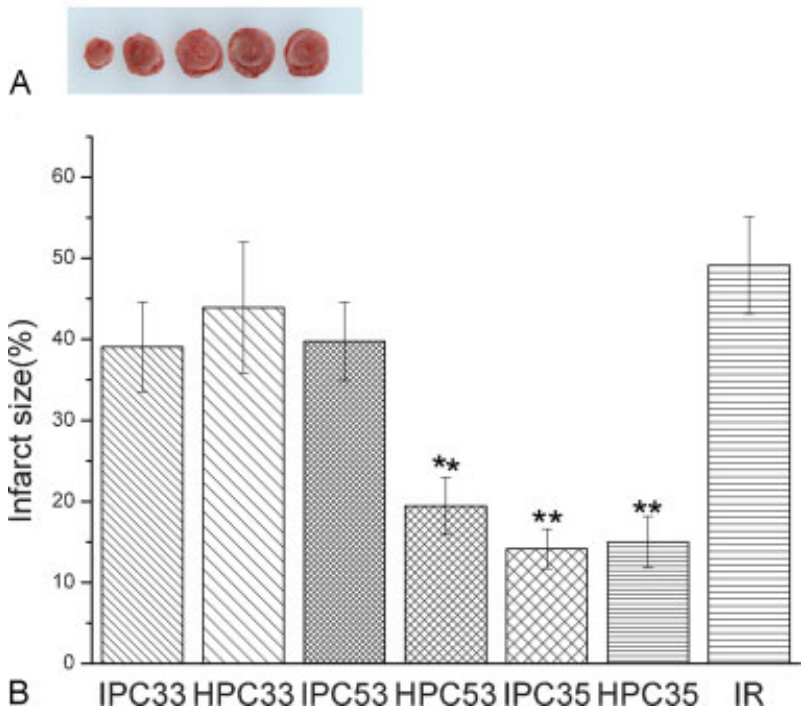

Fig. 3 Effects of IPC and HPC on cardiac infarct size after prolonged ischemia and reperfusion. (A) Histology picture. Sections of hearts were stained with triphenyltetrazolium chloride. (B) Infarct sizes are expressed as a percentage of the total slice area. Data are expressed as mean \pm SE, $n=7 .{ }^{*} P<0.05,{ }^{*} P<0.01$ compared with I/R group. Abbreviations: HPC, hypoxic preconditioning; IPC, ischemic preconditioning.

minute hypoxia followed with 5-minute reperfusion). In our study, increasing number of cycles and reducing hypoxic duration of each cycle, which made HPC safer, were able to provide protective effects on I/R hearts. However, we found that IPC35 rather than IPC53 protected I/R hearts and there was little advantage of more than 3 cycles in IPC. Therefore, multiple-cycle HPC may have more attractive potential for clinical application than IPC.

On one hand, the mechanisms of prolonged HPC and delayed cardioprotection by HPC include upregulation and downregulation of various genes. ${ }^{12}$ Recently, Pan et al reported that the expression of glucose-regulated protein 78 (GRP78) peaked at 24 hours after one-cycle HPC and the upregulation of GRP78 mediated the delayed protection in cultured neonatal rat cardiomyocytes. ${ }^{13}$ Another study demonstrated that the interaction of hypoxia inducible factor- 1 with myocardial inducible nitric oxide synthase gene played an important role in delayed cardioprotection induced by intermittent hypoxia in rat. ${ }^{14}$ On the other hand, there must be intrinsic mechanisms that could be rapidly triggered involved in early cardioprotection by short duration HPC. A further understanding of these mechanisms could contribute 
to minimizing early cardioprotection provided by short duration HPC and developing new treatments against I/R injury in the future.

In conclusion, using an isolated rat heart model, our results demonstrated that multiple-cycle short duration HPC exerted early cardioprotection, which was as powerful as that of IPC. Further studies are needed to investigate the underlying mechanisms.

\section{Funding}

This study was supported by grants from the National Nature Science Foundation of China (81070117).

\section{References}

1 Powers SK, Murlasits Z, Wu M, Kavazis AN. Ischemia-reperfusioninduced cardiac injury: a brief review. Med Sci Sports Exerc 2007;39(9):1529-1536

2 De Hert SG, Preckel B, Hollmann MW, Schlack WS. Drugs mediating myocardial protection. Eur J Anaesthesiol 2009;26(12): 985-995

3 Lin JS, Chen YS, Chiang HS, Ma MC. Hypoxic preconditioning protects rat hearts against ischaemia-reperfusion injury: role of erythropoietin on progenitor cell mobilization. J Physiol 2008;586 (Pt 23):5757-5769

4 Bautista L, Castro MJ, López-Barneo J, Castellano A. Hypoxia inducible factor-2alpha stabilization and maxi-K+ channel beta1-subunit gene repression by hypoxia in cardiac myocytes: role in preconditioning. Circ Res 2009;104(12):1364-1372
5 Mallet RT, Ryou MG, Williams AG Jr, Howard L, Downey HF. Beta1Adrenergic receptor antagonism abrogates cardioprotective effects of intermittent hypoxia. Basic Res Cardiol 2006;101(5):436-446

6 Calmettes G, Deschodt-Arsac V, Gouspillou G, et al. Improved energy supply regulation in chronic hypoxic mouse counteracts hypoxia-induced altered cardiac energetics. PLoS ONE 2010;5(2): e9306

7 Shizukuda Y, Mallet RT, Lee SC, Downey HF. Hypoxic preconditioning of ischaemic canine myocardium. Cardiovasc Res 1992; 26(5):534-542

8 Lasley RD, Anderson GM, Mentzer RM Jr. Ischaemic and hypoxic preconditioning enhance postischaemic recovery of function in the rat heart. Cardiovasc Res 1993;27(4):565-570

9 Mei DA, Nithipatikom K, Lasley RD, Gross GJ. Myocardial preconditioning produced by ischemia, hypoxia, and a KATP channel opener: effects on interstitial adenosine in dogs. J Mol Cell Cardiol 1998;30(6):1225-1236

10 Cai Z, Manalo DJ, Wei G, et al. Hearts from rodents exposed to intermittent hypoxia or erythropoietin are protected against ischemia-reperfusion injury. Circulation 2003;108(1):79-85

11 Zhang SX, Miller JJ, Gozal D, Wang Y. Whole-body hypoxic preconditioning protects mice against acute hypoxia by improving lung function. J Appl Physiol 2004;96(1):392-397

12 Park AM, Nagase H, Vinod Kumar S, Suzuki YJ. Acute intermittent hypoxia activates myocardial cell survival signaling. Am J Physiol Heart Circ Physiol 2007;292(2):H751-H757

13 Pan YX, Ren AJ, Zheng J, et al. Delayed cytoprotection induced by hypoxic preconditioning in cultured neonatal rat cardiomyocytes: role of GRP78. Life Sci 2007;81(13):1042-1049

14 Belaidi E, Beguin PC, Levy P, Ribuot C, Godin-Ribuot D. Prevention of HIF-1 activation and iNOS gene targeting by low-dose cadmium results in loss of myocardial hypoxic preconditioning in the rat. Am J Physiol Heart Circ Physiol 2008;294(2):H901-H908 